\title{
Molecular Epidemiology of Prostate Cancer
}

National Cancer Institute

\section{Source}

National Cancer Institute. Molecular Epidemiology of Prostate Cancer. NCI Thesaurus.

Code C19257.

The objective is to examine the role of genes that regulate the metabolism of environmental carcinogens in prostate cancer etiology. These genes include cytochromes P450 (e.g. CYP1A1, CYP2D6, and CYP2E1) and the mu or theta classes of the glutathione-S-transferases. Three specific aims are proposed to accomplish this objective. In Specific Aim I, the relationship of each of the candidate genes and the occurrence or age of onset of prostate cancer will be examined. The relationship between multiple candidate genes and the occurrence or age of onset of prostate cancer will be examined in Specific Aim 2. The interactions of multiple candidate genes, environments, and exposures will be examined in Specific Aim 3. (from EGP Research Programs, PI: T imothy R. Rebbeck, University of Pennsylvania, R29_ES_08031_03) 